\title{
Long-term outcomes of extracorporeal membrane oxygenation support for postcardiotomy shock
}

\author{
Shao-Wei Chen, MD, ${ }^{\mathrm{a}, \mathrm{b}}$ Feng-Chun Tsai, MD, ${ }^{\mathrm{a}}$ Yu-Sheng Lin, MD, ${ }^{\mathrm{c}}$ Chih-Hsiang Chang, MD, ${ }^{\mathrm{b}, \mathrm{d}}$ \\ Dong-Yi Chen, MD, ${ }^{\mathrm{e}}$ An-Hsun Chou, MD, PhD, ${ }^{\mathrm{f}}$ and Tien-Hsing Chen, $\mathrm{MD}^{\mathrm{g}}$
}

\section{ABSTRACT}

Objective: Extracorporeal membrane oxygenation (ECMO) is a widely used technique for treating postcardiotomy cardiogenic shock (PCS); however, no study has compared the long-term outcomes of patients who receive ECMO support for PCS with those of the general population post cardiac surgery.

Methods: A total of 1141 patients who received ECMO after cardiac surgery between 2000 and 2011 were identified by using the Taiwan National Health Insurance Research Database. For each patient, we matched 5 non-ECMO patients who had undergone cardiac surgery by using propensity scores calculated for age, sex, 12 comorbid diseases, Charlson score, hospital level, type of cardiac surgery, and year of index hospitalization. The outcomes included all-cause mortality, readmission for any cause, and medical expenditure.

Results: The incidence of ECMO use after cardiac surgery in Taiwan was $1.91 \%$. The ECMO group had a significantly higher risk of in-hospital mortality than did the non-ECMO group $(61.7 \%$ vs $6.8 \%$, odds ratio $22.34,95 \%$ confidence interval 19.06-26.18). The risks of all-cause mortality and first readmission for any cause were greater in the ECMO group than that in the control group $(P<.001$, $P<.001)$ in the first year, whereas no difference was observed after the first year of follow-up $(P=.209, P=.474)$. Similar results were observed regarding the medical expenditure of admission after index admission discharge.

Conclusions: Patients receiving ECMO for PCS had similar outcomes to those of the non-ECMO group after the first year of follow-up despite significantly poor outcomes during the in-hospital course. ( $\mathrm{J}$ Thorac Cardiovasc Surg 2017;154:469-77)

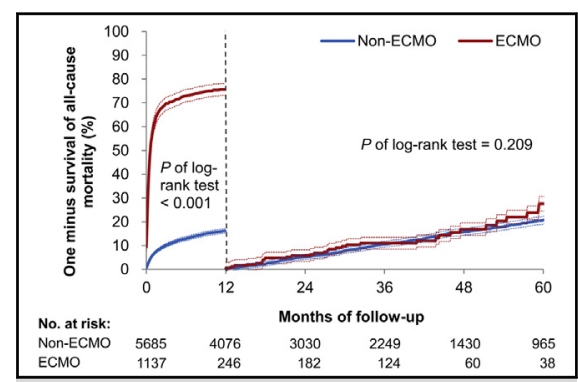

Mortality after cardiac surgery in patients who did or did not receive ECMO support.

\section{Central Message}

Patients receiving ECMO for PCS had equal outcomes to those of a non-ECMO group after the first year of follow-up.

\section{Perspective}

ECMO for PCS had equal outcomes, including mortality, readmission for any cause, and medical expenditure, to those of a control group after the first year of follow-up. ECMO might provide favorable long-term prognosis in selected populations. Acute renal failure and massive blood transfusion are identified as risk factors for in-hospital mortality.

See Editorial Commentary page 478.
Approximately $1 \%$ of adult patients who undergo cardiac surgery develop profound postcardiotomy cardiogenic shock (PCS) and require temporary mechanical circulatory support

\footnotetext{
From the ${ }^{\mathrm{a}}$ Division of Thoracic and Cardiovascular Surgery, Department of Surgery, Chang Gung Memorial Hospital, Linkou Medical Center, Chang Gung University; ${ }^{\mathrm{b}}$ Graduate Institute of Clinical Medical Sciences, College of Medicine, Chang Gung University; 'Department of Cardiology, Chang Gung Memorial Hospital, Chiayi Branch, Chiayi City; ${ }^{\mathrm{d}}$ Kidney Research Center, Department of Nephrology, and ${ }^{\mathrm{e}}$ Department of Cardiology, Chang Gung Memorial Hospital, Linkou Medical Center; ${ }^{\mathrm{f}}$ Department of Anesthesiology, Chang Gung Memorial Hospital, Chang Gung University, Taoyuan City; and ${ }^{\mathrm{g}}$ Department of Cardiology, Chang Gung Memorial Hospital, Keelung Branch, Keelung, Taiwan.

Received for publication May 25, 2016; revisions received Jan 28, 2017; accepted for publication Feb 27, 2017; available ahead of print April 13, 2017.

Address for reprints: Tien-Hsing Chen, MD, Department of Cardiology, Chang Gung Memorial Hospital, Keelung, No. 5 Fuxing St, Guishan District, Taoyuan City 33305, Taiwan (E-mail: skyheart0826@gmail.com or 8702027@cgmh.org.tw). $0022-5223 / \$ 36.00$

Copyright (c) 2017 by The American Association for Thoracic Surgery http://dx.doi.org/10.1016/j.jtcvs.2017.02.055
}

(MCS). ${ }^{1}$ Extracorporeal membrane oxygenation (ECMO), because of its easy and rapid applicability, is widely used for MCS; the technique can facilitate myocardial function recovery and serve as a bridge to further therapy. ${ }^{2-4}$ ECMO, which may constitute the last line of therapy for critically ill patients viewed as unlikely to survive without prompt MCS, has been reported as bringing positive outcomes in selected patients. ${ }^{5-9}$ However, ECMO support after cardiac surgery carries a high mortality rate of $40 \%$ to $80 \%, 1,6,10,11$ and severe morbidity risks, particularly

Scanning this QR code will take you to the supplemental tables, figure, and video for this article.

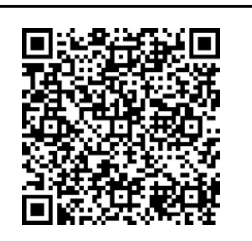




\section{Abbreviations and Acronyms

\begin{tabular}{|c|c|}
\hline CABG & $=$ coronary artery bypass grafting \\
\hline CCI & $=$ Charlson comorbidity index \\
\hline $\mathrm{CI}$ & $=$ confidence interval \\
\hline ECMO & $\begin{aligned}= & \text { extracorporeal membrane } \\
& \text { oxygenation }\end{aligned}$ \\
\hline MCS & $\begin{array}{l}=\text { temporary mechanical circulatory } \\
\text { support }\end{array}$ \\
\hline IABP & $=$ intra-aortic balloon pump \\
\hline ICD-9. & $\begin{aligned} \text { I }= & \text { International Classification of } \\
& \text { Diseases, Ninth Revision, Clinical } \\
& \text { Modification }\end{aligned}$ \\
\hline NHIRD & $\begin{aligned}= & \text { National Health Insurance Research } \\
& \text { Database }\end{aligned}$ \\
\hline DR & $=$ odds ratio \\
\hline & $=$ postcardiotomy cardiogenic shock \\
\hline
\end{tabular}

bleeding and thromboembolic complications. ${ }^{12}$ Furthermore, a paucity of data on the long-term follow-up and detailed outcomes of ECMO for PCS raises concern regarding unwarranted use of ECMO and futile medical treatment.

In Taiwan, the use of ECMO for PCS is recommended, and the associated medical expenditure is reimbursed under the National Health Insurance (NHI) program. Therefore, ECMO is liberally used for PCS support without consideration of the financial costs. Thus, most patients in Taiwan who experience PCS receive ECMO support. Many retrospective and observational studies have demonstrated that the use of ECMO in Taiwan is effective. ${ }^{2,8,13-15}$ Nearly the entire population of Taiwan has been covered by the NHI program since 1995; thus, the NHI claims database provides a unique platform for the detailed review and long-term follow-up of patients after they receive ECMO support. ${ }^{16}$

No previous study has reported the relative outcomes of patients who receive ECMO support for PCS compared with the general population post cardiac surgery without PCS by using a nationwide database. Therefore, we conducted a population-based cohort study to investigate the mortality, complications, readmissions, and medical expenditure of patients who received ECMO support for PCS compared with a matched cohort during a 12-year period in Taiwan.

\section{MATERIALS AND METHODS Data Source}

This study was performed using data collected under the governmentoperated single-payer NHI program, which was launched in 1995 and covered nearly all $(99 \%)$ of the approximately 23 million residents of Taiwan as of December 2015. ${ }^{17}$ The National Health Insurance Research Database (NHIRD) is derived from the NHI program, which reimburses the costs of all lifesaving procedures, surgeries, and medication, including cardiac surgery.

\section{Study Population}

This was an open, observational, longitudinal cohort study comparing the outcomes of patients who did and did not receive ECMO support after cardiac surgery. We defined cardiac surgery as either a coronary artery bypass grafting (CABG) or valve surgery, the 2 most common types of heart surgery. We examined the NHIRD hospitalization records of all patients admitted for cardiac surgery between January 2000 and December 2011. The patients were identified according to the presence of an NHI procedure code for valve repair or replacement $(68,015,68,016,68,017$, and $68,018)$ or for CABG $(68,023,68,024$, and 68,025$)$. In total, 68,186 patients who underwent either surgery during the study period in Taiwan were identified. Patients were excluded if they were younger than 18 years or had missing demographic or surgical information. Patients with repeat hospitalizations for cardiac surgery and concomitant aortic surgery during the index hospitalization also were excluded. International Classification of Diseases, Ninth Revision, Clinical Modification (ICD-9-CM) procedure code 39.65 was used to extract patients who received ECMO. The database does not permit definitive knowledge of the exact timing of ECMO implantation during index admission; however, we presumed that if a patient underwent ECMO and cardiac surgery during the same admission, most ECMO implantation occurred after cardiac surgery. Moreover, we validated the study design by using our own hospital database and, according to our study design, only $1.9 \%$ of ECMO took place before cardiac surgery. This was thus a rare occurrence that accounted for a small percentage of instances and was unlikely to affect our result.

\section{Comorbidities and Outcomes}

For all patients, we obtained inpatient records for at least 3 years before the index admission to track their history of comorbidities. For example, we enrolled patients in 2000 and identified comorbidities from their medical records since 1997. The hospitalization records from this period were used to identify preoperative comorbidities. The baseline characteristics and surgical details of the patients were identified using ICD-9-CM and NHI procedure codes for the index hospitalization and prior hospitalizations (Table E1). The accuracy of the ICD-9-CM, NHI procedure codes, and identification of the comorbidities of NHIRD has been validated in patients with cardiovascular disease and cardiac surgery; the NHIRD appears to be a valid resource for population research. ${ }^{18,19}$ The outcomes of interest in this study included ECMOrelated complications, all-cause mortality, first readmission and cumulative readmissions for any cause, and medical expenditure of admission. Withdrawal from the NHI program was used to identify mortality. ${ }^{20}$ Inhospital expenditure was directly collected from the NHIRD. The date on which a patient was admitted for cardiac surgery was defined as the index hospitalization. The patients were followed from their index admission to December 31, 2011, or death; no missing data were found during the follow-up period in the database.

\section{Propensity Score Matching}

The retrospective study design did not allow the random allocation of the patients; this might raise a concern of selection bias. Therefore, we performed propensity score matching to compare patients who received ECMO with those who did not receive ECMO. ${ }^{21}$ Each patient who received ECMO was matched with 5 patients who did not receive ECMO by using propensity scores according to age, sex, comorbidities, Charlson comorbidity index (CCI), hospital level (Table 1), surgical characteristics (Table 2), and index admission year. We adopted the greedy nearest neighbor matching algorithm and set the maximum tolerance level as 0.2 times the standard deviation of the propensity score (briefly, caliper $=0.2$ ) to guarantee the matching quality. An absolute standardized difference of less than 0.1 after matching was considered to indicate well-balanced matching variables between the groups. ${ }^{22}$ Finally, 1141 patients who received ECMO were included before matching, and 4 of them were excluded after matching, yielding 
a total number of 1137 patients in the ECMO group and 5685 in the non-ECMO group (Figure 1).

\section{Statistical Analysis}

We compared the clinical and surgical characteristics of the ECMO and non-ECMO groups by using the $\chi^{2}$ and $t$ tests for categorical and continuous variables (eg, age), respectively. The risks of in-hospital complications and outcomes between the study groups after matching were compared by using a univariate logistic regression model in which study group was the only covariate. Continuous outcomes (ie, intensive care unit [ICU] days, hospital stays, and inpatient medical expenditure) in the ECMO and non-ECMO groups after matching were compared by using a Mann-Whitney $U$ test. We performed multivariable logistic regression analysis to evaluate the association of post-ECMO complications with the risk of in-hospital mortality. We adjusted for all the covariates listed in Table 1 and surgical type, and the 7 post-ECMO complications (eg, ischemic stroke and acute renal failure) were introduced into the multivariable logistic model simultaneously. To compare the risk of all-cause mortality between the study groups, we constructed survival curves by using the Kaplan-Meier method in addition to the log rank test. For conducting comparisons among the groups with regard to time-to-event outcomes other than mortality (eg, first readmission for any cause), we performed competing risk survival analysis, in which mortality was considered a competing risk. ${ }^{23}$ With respect to comparing the mean number of hospitalizations between the groups, we applied the generalized linear mixed model in which the Poisson distribution was set and the logarithm of follow-up duration was treated as an offset variable; moreover, the effect of multiple events involving a patient was considered in the model. We stratified the patients in the ECMO group by age, $\mathrm{CCI}$, and surgery type and then constructed Kaplan-Meier survival curves of all-cause mortality along with the log rank test to compare differences. In addition, risk factors of 1-year mortality in the ECMO group were investigated by using a multivariable Cox proportional hazard model in which all the variables listed in Table 1 and surgical type were treated as covariates. Medical expenditure (cost for all subsequent hospital admissions) after the index admission discharge were compared between the study groups by using the MannWhitney $U$ test. The medical expenditures from 2000 to 2010 were normalized to 2011 according to the consumer price index of Taiwan. All data analyses were conducted by using SPSS 22 (IBM Corp, Armonk, NY).

\section{RESULTS}

Study Population Characteristics

Table 1 shows the baseline demographics and comorbidities of both study groups. We identified 1141 patients who received ECMO support for PCS between 2000 and 2011 and who met the inclusion criteria. These patients had a mean age of $63.8 \pm 13.2$ years, and $71.3 \%$ of them were men. The incidence of ECMO use after cardiac surgery was $1.91 \%$. Table 2 presents the cardiac surgery history

TABLE 1. Demographic and clinical characteristics of the ECMO and non-ECMO groups

\begin{tabular}{|c|c|c|c|c|c|c|c|c|}
\hline \multirow[b]{2}{*}{ Variable } & \multicolumn{4}{|c|}{ Before matching } & \multicolumn{4}{|c|}{ After matching } \\
\hline & ECMO & Non-ECMO & $P$ & SMD & ECMO & Non-ECMO & $P$ & SMD \\
\hline Patient number & 1141 & 58,579 & N/A & N/A & 1137 & 5685 & N/A & N/A \\
\hline Age, y & $63.8 \pm 13.2$ & $63.3 \pm 12.8$ & .213 & 0.04 & $63.8 \pm 13.2$ & $63.7 \pm 13.3$ & .765 & 0.01 \\
\hline Age group, y & & & .005 & & & & .986 & \\
\hline$<40$ & $59(5.2)$ & $2918(5.0)$ & & 0.01 & $59(5.2)$ & $306(5.4)$ & & -0.01 \\
\hline $41-50$ & $108(9.5)$ & $5724(9.8)$ & & -0.01 & $108(9.5)$ & $570(10.0)$ & & -0.02 \\
\hline $51-60$ & 244 (21.4) & $12,628(21.6)$ & & 0.00 & $242(21.3)$ & $1171(20.6)$ & & 0.02 \\
\hline $61-70$ & 315 (27.6) & $17,006(29.0)$ & & -0.03 & 315 (27.7) & $1554(27.3)$ & & 0.01 \\
\hline $71-80$ & $308(27.0)$ & $16,552(28.3)$ & & -0.03 & 306 (26.9) & $1539(27.1)$ & & 0.00 \\
\hline$>80$ & $107(9.4)$ & $3751(6.4)$ & & 0.11 & $107(9.4)$ & $545(9.6)$ & & -0.01 \\
\hline Gender & & & .187 & & & & .553 & \\
\hline Male & $813(71.3)$ & $40,675(69.4)$ & & 0.04 & $810(71.2)$ & $4000(70.4)$ & & 0.02 \\
\hline Female & $328(28.7)$ & $17,904(30.6)$ & & -0.04 & 327 (28.8) & 1685 (29.6) & & -0.02 \\
\hline \multicolumn{9}{|l|}{ Comorbid conditions } \\
\hline Diabetes mellitus & $434(38.0)$ & $22,725(38.8)$ & .603 & -0.02 & $432(38.0)$ & 2157 (37.9) & .973 & 0.00 \\
\hline Hypertension & $624(54.7)$ & $35,186(60.1)$ & $<.001$ & -0.11 & $622(54.7)$ & $3098(54.5)$ & .896 & 0.00 \\
\hline Congestive heart failure & $308(27.0)$ & $13,714(23.4)$ & .005 & 0.08 & $307(27.0)$ & $1533(27.0)$ & .981 & 0.00 \\
\hline Coronary artery disease & $937(82.1)$ & $45,758(78.1)$ & .001 & 0.10 & $934(82.1)$ & $4659(82.0)$ & .877 & 0.01 \\
\hline Prior myocardial infarction & $253(22.2)$ & $10,451(17.8)$ & $<.001$ & 0.11 & $252(22.2)$ & $1256(22.1)$ & .958 & 0.00 \\
\hline Peripheral arterial disease & $65(5.7)$ & $2675(4.6)$ & .071 & 0.05 & $65(5.7)$ & $312(5.5)$ & .758 & 0.01 \\
\hline Prior stroke & $174(15.2)$ & $7520(12.8)$ & .016 & 0.07 & $173(15.2)$ & $858(15.1)$ & .916 & 0.00 \\
\hline Chronic kidney disease & $124(10.9)$ & $4013(6.9)$ & $<.001$ & 0.14 & $124(10.9)$ & $660(11.6)$ & .497 & -0.02 \\
\hline Dialysis & $83(7.3)$ & $2349(4.0)$ & $<.001$ & 0.14 & $83(7.3)$ & $436(7.7)$ & .668 & -0.01 \\
\hline COPD & $89(7.8)$ & $4745(8.1)$ & .713 & -0.01 & $89(7.8)$ & $460(8.1)$ & .765 & -0.01 \\
\hline Liver cirrhosis & $22(1.9)$ & $795(1.4)$ & .100 & 0.04 & $22(1.9)$ & $107(1.9)$ & .905 & 0.00 \\
\hline Malignancy & $39(3.4)$ & $1873(3.2)$ & .675 & 0.01 & $39(3.4)$ & 204 (3.6) & .793 & -0.01 \\
\hline Charlson score & $2.6 \pm 2.2$ & $2.0 \pm 2.0$ & $<.001$ & 0.27 & $2.6 \pm 2.2$ & $2.6 \pm 2.5$ & .956 & 0.00 \\
\hline Hospital level & & & $<.001$ & & & & .849 & \\
\hline Medical center (teaching) & $725(63.5)$ & $39,982(68.3)$ & & -0.10 & $724(63.7)$ & $3647(64.2)$ & & -0.01 \\
\hline Regional hospital & $402(35.2)$ & $18,357(31.3)$ & & 0.08 & $399(35.1)$ & $1978(34.8)$ & & 0.01 \\
\hline District hospital (local) & $14(1.2)$ & $240(0.4)$ & & 0.09 & $14(1.2)$ & $60(1.1)$ & & 0.02 \\
\hline
\end{tabular}

$E C M O$, Extracorporeal membrane oxygenation; $S M D$, standardized mean difference; $C O P D$, chronic obstructive pulmonary disease; N/A, not applicable. 
TABLE 2. Surgical characteristics of the ECMO and non-ECMO groups

\begin{tabular}{|c|c|c|c|c|c|c|c|c|}
\hline \multirow[b]{2}{*}{ Variable } & \multicolumn{4}{|c|}{ Before matching } & \multicolumn{4}{|c|}{ After matching } \\
\hline & $\begin{array}{c}\text { ECMO } \\
(n=1141)\end{array}$ & $\begin{array}{l}\text { Non-ECMO } \\
(\mathrm{n}=\mathbf{5 8 , 5 7 9})\end{array}$ & $P$ & SD & $\begin{array}{c}\text { ECMO } \\
(n=1137)\end{array}$ & $\begin{array}{c}\text { Non-ECMO } \\
(\mathbf{n}=\mathbf{5 6 8 5})\end{array}$ & $P$ & SD \\
\hline Surgical type & & & $<.001$ & & & & .797 & \\
\hline CABG alone & $729(63.9)$ & $36,964(63.1)$ & & 0.02 & $728(64.0)$ & $3580(63.0)$ & & 0.02 \\
\hline Valve alone & $275(24.1)$ & $16,697(28.5)$ & & -0.10 & 275 (24.2) & $1416(24.9)$ & & -0.02 \\
\hline CABG + Valve & $137(12.0)$ & $4918(8.4)$ & & 0.12 & 134 (11.8) & $689(12.1)$ & & -0.01 \\
\hline \multicolumn{9}{|l|}{ CABG details } \\
\hline Number of bypass vessels & & & .049 & & & & .951 & \\
\hline 1 & $134(15.5)$ & $5364(12.8)$ & & 0.08 & $133(15.4)$ & $681(16.0)$ & & -0.01 \\
\hline 2 & $275(31.8)$ & $13,895(33.2)$ & & -0.03 & $274(31.8)$ & $1318(30.9)$ & & 0.02 \\
\hline 3 & $348(40.2)$ & $16,480(39.3)$ & & 0.02 & $346(40.1)$ & $1731(40.5)$ & & -0.01 \\
\hline 4 or more & 109 (12.6) & 6143 (14.7) & & -0.06 & 109 (12.6) & 539 (12.6) & & 0.00 \\
\hline Type of CABG & & & $<.001$ & & & & .892 & \\
\hline On pump & $562(64.9)$ & $33,980(81.1)$ & & -0.37 & $561(65.1)$ & $2768(64.8)$ & & 0.01 \\
\hline Off pump & $304(35.1)$ & 7902 (18.9) & & 0.37 & $301(34.9)$ & $1501(35.2)$ & & -0.01 \\
\hline Endarterectomy & $66(7.6)$ & $1532(3.7)$ & $<.001$ & 0.17 & $63(7.3)$ & $282(6.6)$ & .452 & 0.03 \\
\hline \multicolumn{9}{|l|}{ Valve surgery details } \\
\hline Number of involved valves & & & .484 & & & & .954 & \\
\hline 1 & $278(67.5)$ & $14,119(65.3)$ & & 0.05 & $277(67.7)$ & $1420(67.5)$ & & 0.01 \\
\hline 2 & 119 (28.9) & $6475(30.0)$ & & -0.02 & 117 (28.6) & $601(28.6)$ & & 0.00 \\
\hline 3 & $15(3.6)$ & $1021(4.7)$ & & -0.05 & $15(3.7)$ & $84(4.0)$ & & -0.02 \\
\hline \multicolumn{9}{|l|}{ Type of valve surgery } \\
\hline Aortic valve repair or replacement & $163(39.6)$ & $9369(43.3)$ & .125 & -0.08 & $162(39.6)$ & $843(40.0)$ & .868 & -0.01 \\
\hline Mitral valve repair & $96(23.3)$ & $5325(24.6)$ & .533 & -0.03 & $95(23.2)$ & $509(24.2)$ & .680 & -0.02 \\
\hline Mitral valve replacement & $205(49.8)$ & 9499 (43.9) & .019 & 0.12 & $202(49.4)$ & $1032(49.0)$ & .893 & 0.01 \\
\hline Tricuspid repair & $79(19.2)$ & $5293(24.5)$ & .013 & -0.13 & $79(19.3)$ & $404(19.2)$ & .954 & 0.00 \\
\hline Tricuspid replacement & $21(5.1)$ & $610(2.8)$ & .006 & 0.12 & $20(4.9)$ & $102(4.8)$ & .970 & 0.00 \\
\hline Pulmonary valve repair/replacement & $1(0.2)$ & $138(0.6)$ & .315 & -0.06 & $1(0.2)$ & $5(0.2)$ & .979 & 0.00 \\
\hline
\end{tabular}

$E C M O$, Extracorporeal membrane oxygenation; $S D$, standardized difference; $C O P D$, chronic obstructive pulmonary disease.

of the study groups before and after propensity score matching. After matching, the absolute standardized difference of each variable was less than 0.1 , indicating the distribution of matching variables was well-balanced between groups.

\section{In-Hospital Mortality and Postoperative \\ Complications}

The ECMO group had a significantly higher risk of inhospital mortality than did the non-ECMO group $(61.7 \%$ vs $6.8 \%$, odds ratio $22.34,95 \%$ confidence interval [CI] 19.06-26.18). The ECMO group also had a higher risk of postoperative complications than did the non-ECMO group, including reexploration for bleeding, massive blood transfusion with more than 8 units of packed red blood cells, new-onset hemorrhagic stroke, acute renal failure requiring hemodialysis, postoperative infection, leg ischemia requiring fasciotomy or amputation and intra-aortic balloon pump (IABP) support. In addition, the inpatient medical expenditure of the ECMO group was approximately twice that of the non-ECMO group (median: 76.7 vs $38.5 \mathrm{New}$ Taiwan Dollar [NTD] $\left.\times 10^{4}, P<.001\right)$. In addition, the patients in the ECMO group had longer ICU durations, but slightly shorter hospital stays (Table 3).
In addition, the following variables were found be associated with a higher chance of survival during the index admission in the ECMO group: a younger age, presence of hypertension, absence of heart failure, and absence of liver cirrhosis (Table E2).

\section{All-Cause Mortality, First Readmission, Cumulative Readmission, and Medical Expenditure During Follow-up}

Compared with the non-ECMO group, the ECMO group had a lower Kaplan-Meier survival rate during follow-up for 1 year $(24.1 \%$ [95\% CI, 21.6-26.6] vs 83.4\% [95\% CI, $82.4-84.4]), 5$ years $(17.7 \% \quad[95 \%$ CI, 14.7-20.7] vs $66.0 \%$ [95\% CI, 64.3-67.6]), and 10 years $(9.7 \%$ [95\% CI, 4.0-15.5] vs 50.2\% [95\% CI, 46.7-53.7]).

Figure 2, $A$ and $B$, illustrate the Kaplan-Meier survival curves for all-cause mortality and first readmission for any cause, respectively. The results revealed that the risk of all-cause mortality was greater in the ECMO group than in the non-ECMO group $(P<.001)$ in the first year of follow-up, whereas no difference was observed after the first year of follow-up $(P=.209$; Figure $2, A)$. Similar results were observed regarding first readmission for any 


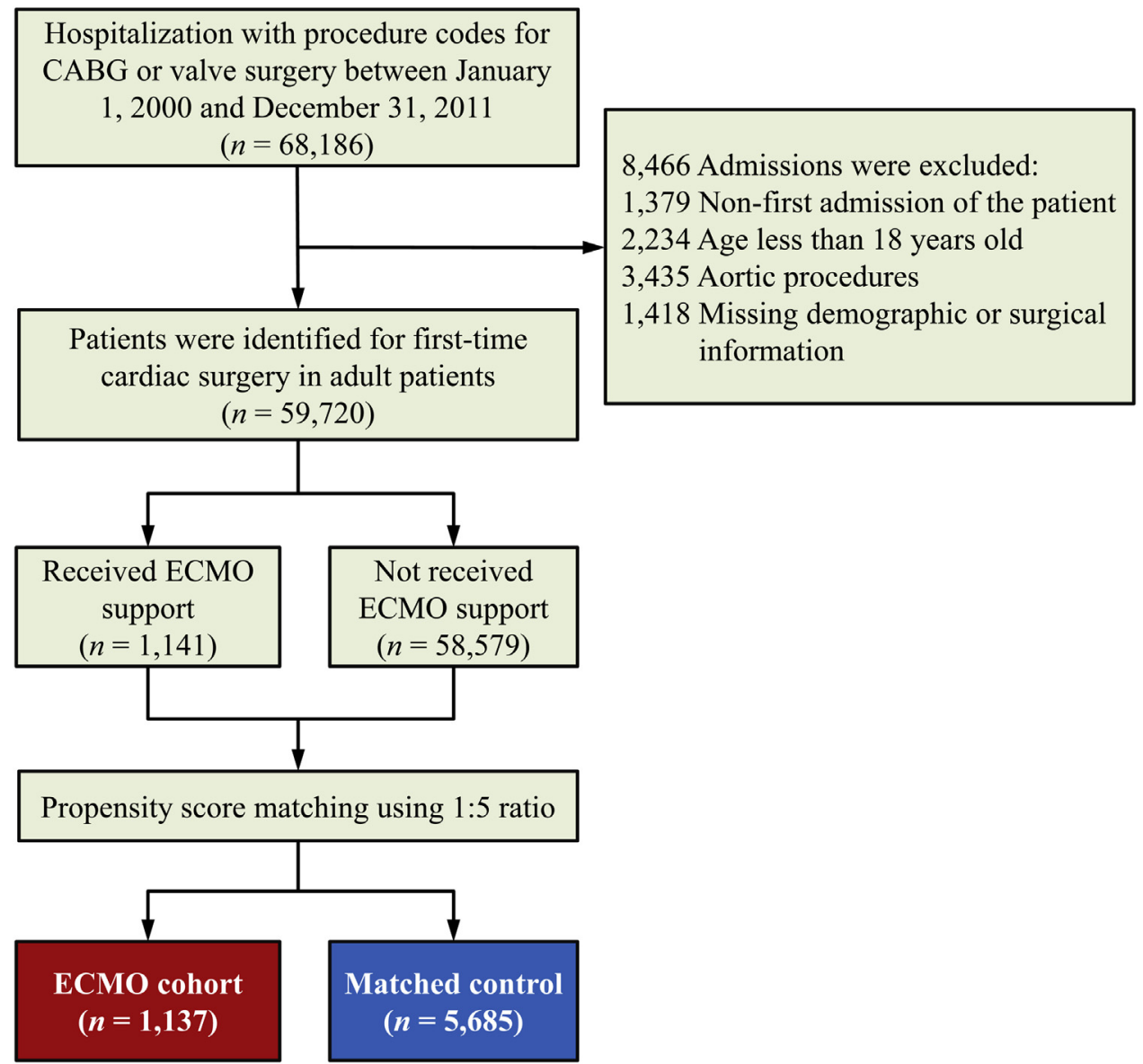

FIGURE 1. Enrollment of the population-based cohort of 68,186 adult patients who underwent first-time CABG or valve surgery between 2000 and 2011 , extracted from the Taiwan NHIRD. $C A B G$, Coronary artery bypass grafting; ECMO, extracorporeal membrane oxygenation.

cause (Figure 2, B). We also observed a greater mean number of readmissions for any cause during the follow-up in the ECMO group (mean, $0.68 ; 95 \%$ CI, 0.60-0.78) than in the non-ECMO group (mean, $0.52 ; 95 \% \mathrm{CI}, 0.50-0.55$ ) and this group difference was statistically significant (rate ratio [RR] 1.31; 95\% CI, 1.13-1.50; Figure 2, C).
Figure 3 presents the medical expenditure (cost for all subsequent hospital admissions) after index admission discharge. This analysis included those patients who were followed and survived beyond the specific follow-up period (ie, the first year). The results revealed that medical expenditure was greater for the ECMO group than for the non-

TABLE 3. Surgery-related complications and outcomes

\begin{tabular}{|c|c|c|c|c|}
\hline Variable & $\operatorname{ECMO}(n=1137)$ & Non-ECMO $(n=5685)$ & $\begin{array}{c}\text { ECMO vs Non-ECMO } \\
\text { OR/B }(95 \% \text { CI })\end{array}$ & $\boldsymbol{P}$ \\
\hline In-hospital mortality & $701(61.7)$ & $385(6.8)$ & $22.34(19.06-26.18)$ & $<.001$ \\
\hline Reexploration for bleeding & $129(11.3)$ & $141(2.5)$ & $5.04(3.93-6.45)$ & $<.001$ \\
\hline Massive blood transfusion, PRBC $>8$ Units & $899(79.1)$ & $870(15.3)$ & $21.25(18.09-24.96)$ & $<.001$ \\
\hline New-onset ischemic stroke & $36(3.2)$ & $201(3.5)$ & $0.89(0.62-1.28)$ & .534 \\
\hline New-onset hemorrhagic stroke & $12(1.1)$ & $23(0.4)$ & $2.63(1.30-5.29)$ & .007 \\
\hline Acute renal failure and need hemodialysis & $374(32.9)$ & $418(7.4)$ & $6.26(5.34-7.35)$ & $<.001$ \\
\hline Postoperative infection & $150(13.2)$ & $256(4.5)$ & $3.23(2.61-4.00)$ & $<.001$ \\
\hline Fasciotomy or amputation & $26(2.3)$ & $47(0.8)$ & $2.81(1.73-4.56)$ & $<.001$ \\
\hline IABP & $728(64.0)$ & $875(15.4)$ & $9.92(8.61-11.43)$ & $<.001$ \\
\hline ICU duration, $\mathrm{d}$ & $9[4,18]$ & $4[3,8]$ & $5.82(5.13,6.51)$ & $<.001^{*}$ \\
\hline Hospital stays, d & $16[7,30]$ & $18[13,27]$ & $-1.13(-2.04,-0.22)$ & $.015^{*}$ \\
\hline Inpatient medical expenditure $\left(\mathrm{NTD} \times 10^{4}\right)$ & $76.7[56.7,104.8]$ & $38.5[30.9,50.8]$ & $41.87(40.12,43.61)$ & $<.001^{*}$ \\
\hline
\end{tabular}



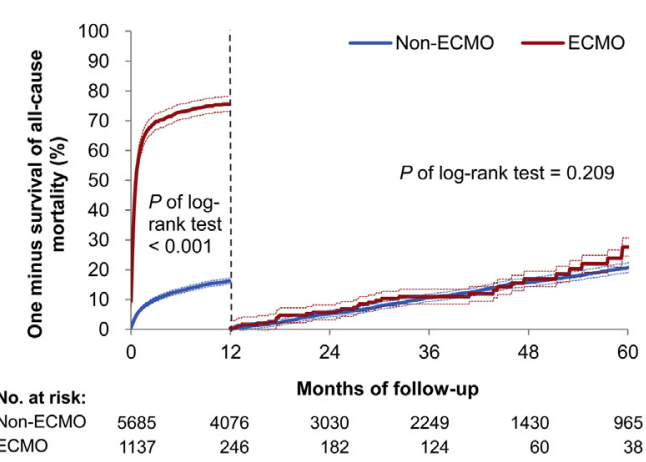
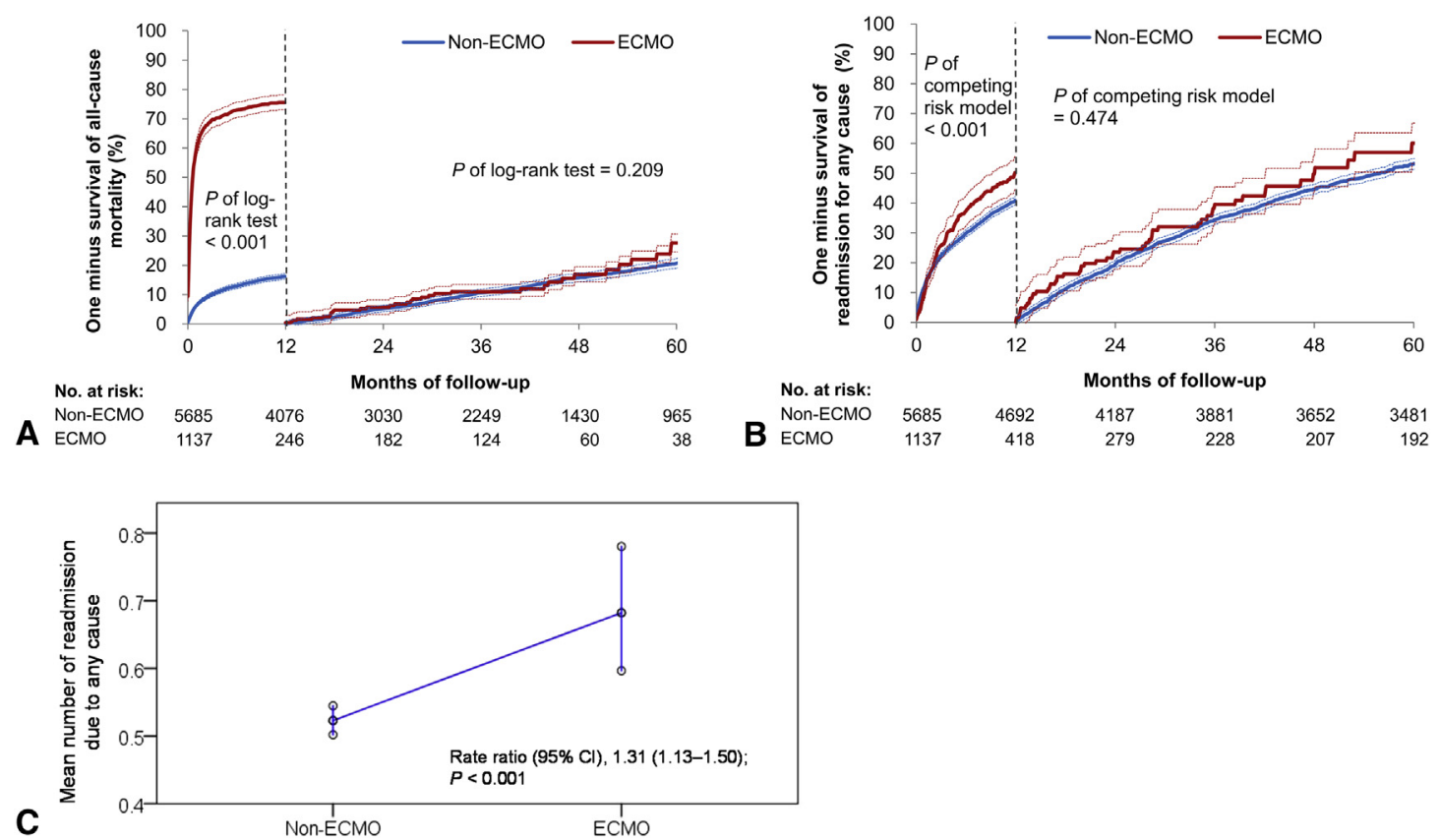

FIGURE 2. Kaplan-Meier survival curves of (A) all-cause mortality and (B) readmission for any cause after cardiac surgery in patients who did or did not receive ECMO support. (C) Mean number of readmissions due to any cause during the follow-up between the groups. ECMO, Extracorporeal membrane oxygenation.

ECMO group during the first year of follow-up $(P<.001)$. No difference between groups was observed from the second year of follow-up onward.

In addition, the following variables were found be associated with a higher chance of survival during the first year of follow-up in the ECMO group: a younger age, absence of heart failure, and absence of chronic kidney disease or dialysis (Table E3).

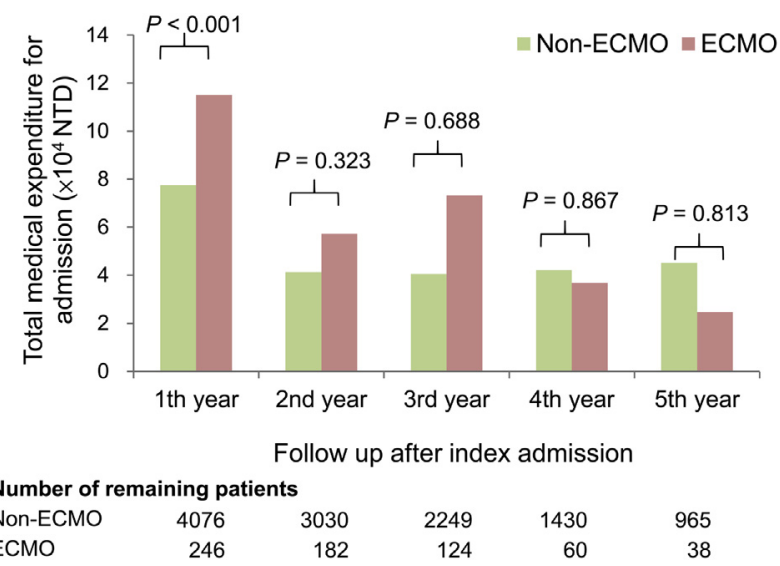

FIGURE 3. Total medical expenditure for admission after the index admission in patients who did or did not receive ECMO support after cardiac surgery. ECMO, Extracorporeal membrane oxygenation; NTD, New Taiwan dollar.

\section{Effect of ECMO-Related Complications on In- Hospital Mortality}

Figure 4 summarizes the results regarding the effects of ECMO-related complications on in-hospital mortality. The results indicated that among the most common ECMO-related complications, acute renal failure requiring hemodialysis (OR 1.94; 95\% CI, 1.45-2.59) and massive blood transfusion with more than 8 units of packed red blood cells (OR 1.58; 95\% CI, 1.15-2.17) were associated with a higher risk of in-hospital mortality.

\section{Associations of Age, Comorbidity, and Surgery Type With Mortality}

Figure E1, $A$ to $C$, shows the Kaplan-Meier survival curves for identifying the correlation of mortality with age, CCI score, and surgery type. The results indicated that an older age and a higher CCI score were associated with a higher all-cause mortality rate (both $P$ for linear trend of $\log$ rank test $<.001$ ). Furthermore, the patients who received both $\mathrm{CABG}$ and valve surgery had a higher risk of mortality during follow-up than did those who received CABG alone or valve surgery alone (both $P$ of log rank test $<.05)$.

\section{DISCUSSION}

In this study, we compared 1137 patients who received ECMO after cardiac surgery in a 12-year period with a matched control group by using a nationwide Taiwan database. The incidence of ECMO use for treating PCS was 


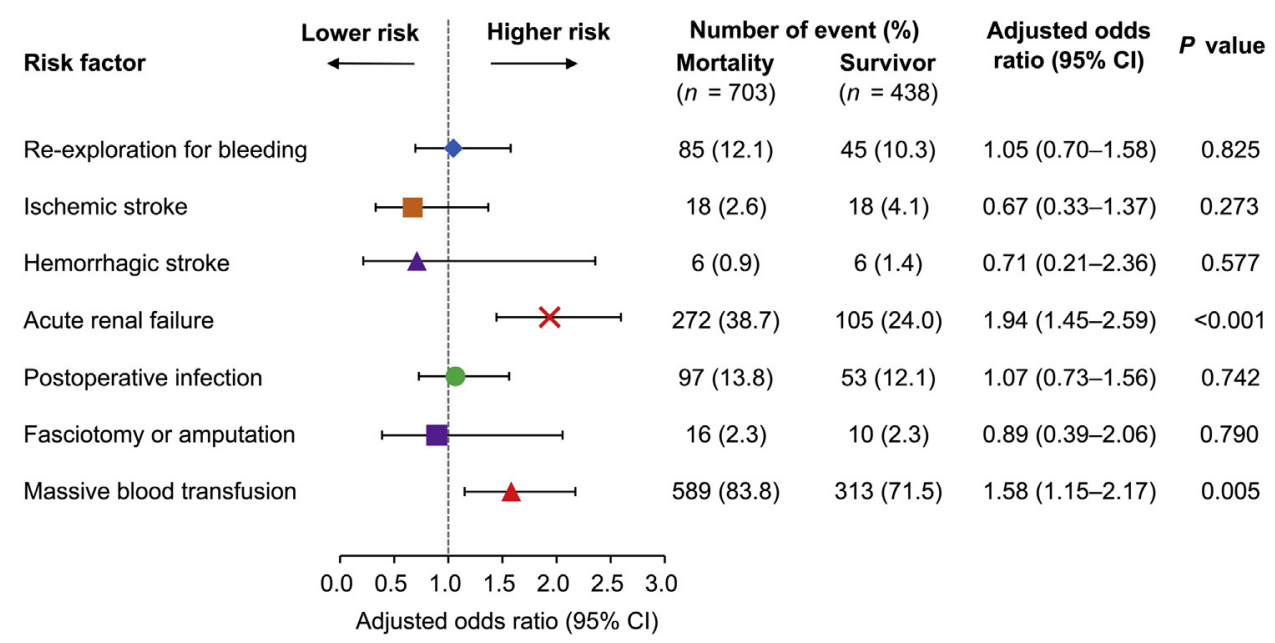

FIGURE 4. Association of ECMO-related complications with risk of in-hospital mortality. This multivariable logistic regression analysis was adjusted for all the variables listed in Table 1 and surgical type in Table 2 and the 7 complications were introduced into the model simultaneously. CI, Confidence interval.

$1.91 \%$, with an in-hospital mortality rate of $61.6 \%$. To our knowledge, this is the largest cohort study of ECMO for PCS. In Taiwan, the NHI sets uniform indications regulated by Taiwan NHI administration for use of ECMO. Furthermore, this is the first study to compare the long-term outcomes of patients who received ECMO support for PCS with those of a matched control group. By doing so, we demonstrated the favorable long-term outcomes after the first year of follow-up for critically ill patients who presumably would not have survived without receiving prompt MCS.

The major findings of this research support the argument that ECMO might provide a favorable late prognosis for patients with PCS. The causes of PCS are complex; in addition to critical preoperative conditions and comorbidities, intraoperative disaster events also contribute to the development of PCS. ${ }^{5}$ Tarzia et $\mathrm{al}^{24}$ demostrated that ECMO alone is associated with a considerable likelihood of recovery from cardiogenic shock with an acute etiology, but not from that with a chronic etiology. The subgroup analysis in our study showed that an older age, advanced preoperative comorbidities, and complex surgery were all related to chronic etiology-associated poor long-term outcomes in the ECMO group. Our results also suggested that ECMO might provide a survival benefit only for patients who experience an intraoperative event and who are in a relatively healthy preoperative condition. Combes et $\mathrm{al}^{25}$ showed that most patients receiving ECMO discharged from ICU could survive at 1 year, and a quality-of-life survey demonstrated a trend toward normalization of quality of life over time after hospitalization. Anselmi et $\mathrm{al}^{26}$ demonstrated that patients who survive acute events as a result of ECMO for cardiopulmonary resuscitation may attain similar health-related quality of life compared with similar populations of critical care patients. Furthermore, the cardiac-RESCUE program of Beurtheret et $\mathrm{al}^{27}$ yielded favorable long-term functional outcomes after ECMO support for refractory cardiogenic shock. Among the patients who received this treatment and were subsequently discharged, the 1-year survival rate was $93.8 \%$, with a New York Heart Association heart failure class of $1.5 \pm 0.6$ during follow-up. However, further investigation in the form of prospective randomized multicenter studies is required to establish how and with whom ECMO should be used.

The use of ECMO for PCS is a lifesaving procedure; however, the literature reports considerable associated morbidity following this intervention, ${ }^{12}$ particularly bleeding and thromboembolism-related complications. Data from this study, which compared an ECMO group with a matched control group, support this notion. No previous study has demonstrated which ECMO-related complications are associated with mortality. We found that massive blood transfusion and acute renal failure are associated with in-hospital mortality. Acute kidney injury requiring renal replacement therapy during ECMO support is associated with poor outcomes. ${ }^{13,28,29}$ Excessive accumulated extravascular water impairs oxygen transport in tissue and is associated with subsequent organ dysfunction. ECMO is associated with bleeding complications and typically requires large blood transfusions. A larger number of transfused red blood cell units is well known to be associated with increased mortality after cardiac surgery, ${ }^{30}$ and this relationship may extend to patients receiving ECMO.$^{31}$ Our study suggests that meticulous hemostasis for avoiding unnecessary bleeding, a restrictive strategy of blood transfusion, and an aggressive renal protective strategy may improve the outcomes of ECMO support for PCS.

\section{Study Strengths}

This study has several strengths. First, the current related literature has a paucity of large randomized trials and is 


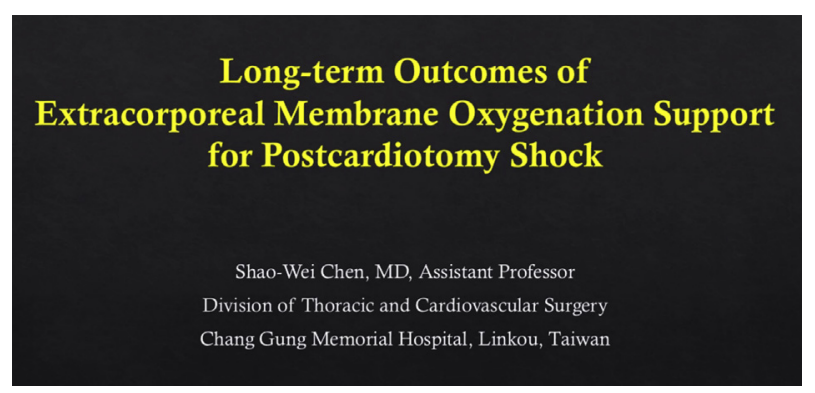

VIDEO 1. The study's lead author speaks about the study's purpose, results, and conclusion of its findings. Video available at: http://www. jtcvsonline.org/article/S0022-5223(17)30417-8/addons.

limited by small sample sizes, single-outcome investigations, and a lack of control groups. We conducted a national observational study of ECMO for PCS and compared outcomes with a non-ECMO group through propensity score matching. The sample size was the largest of any study on this topic, providing adequate statistical power for analyzing variable outcomes. Second, the NHIRD provides a unique platform for the detailed evaluation of outcomes seldom investigated previously, such as readmission and medical expenditure, and enables complete long-term follow-up with limited missing data. Finally, the NHI program covers nearly all Taiwan residents, and ECMO is the only advanced MCS technique covered by the program. Thus, the universal coverage of expensive and advanced ECMO devices reduces socioeconomic or residential location barriers that may have otherwise caused selection bias.

\section{Study Limitations}

Some study limitations should be noted. First, the incidence of ECMO treatment for PCS was relatively high $(1.91 \%)$. It may be due to delayed referral for cardiac surgery and poor preoperative myocardial function being common in Taiwan; these factors may contribute to a higher incidence of PCS. Furthermore, the use of ECMO for PCS is reimbursed under the Taiwan NHI. Therefore, ECMO is liberally used for PCS treatment without consideration of the financial costs, and nearly all patients who experienced severe PCS received ECMO support. However, the NHI program has strict regulations regarding ECMO reimbursements, which are granted only to patients who have clinical or examination evidence of profound PCS. Furthermore, terminal chronic diseases, such as irreversible brain damage, are contraindicated for reimbursement. The second limitation is the relatively high mortality of the control group, which reflects real-world data outside developed Western countries. The low medical expenditure for admissions in Taiwan (approximately 10\% of the costs in the United States) may have negatively affected the outcomes of cardiac surgery. Third, the NHIRD did not include data regarding information such as the ventricular ejection fraction, which is the most powerful predictor of mortality in this population, and other ECMO-related parameters, such as cannula size, insertion (central or peripheral), supporting duration, whether mortality occurred on ECMO or after ECMO removal, laboratory data, or other detailed clinical information reflecting the severity of patients on ECMO implantation, and whether adequate perfusion was achieved. However, we adopted alternative variables as proxies for unavailable information, such as congestive heart failure, which is associated with the left ventricular ejection fraction. Other ECMO parameters are relatively less predictive of long-term outcomes. Finally, the data we claimed included only all inpatient costs. The medical expenditure of the outpatient department was not available in our study. Underestimation of the financial burden by limiting the analysis to hospital expenses only should be noted and evaluated. Despite these limitations, we believe that the strengths of our study provide a valuable contribution to outcome analysis in patients with ECMO for PCS.

\section{CONCLUSIONS}

Compared with the non-ECMO group, the ECMO group had significantly poorer outcomes during the first year of follow-up. However, the all-cause mortality, readmission for any cause, and medical expenditure of the groups did not differ significantly after 1 year of follow-up. We concluded that ECMO support for PCS might yield favorable long-term outcomes (Video 1).

Among the patients who received ECMO support for PCS, we observed that ECMO-associated complications of acute renal failure and massive blood transfusion were risk factors for in-hospital mortality. An older age, advanced preoperative comorbidities, and surgical complexity were associated with long-term mortality. Our study suggests that ECMO might provide a survival benefit only for patients who experience an intraoperative event and who are in a relatively healthy preoperative condition.

\section{Conflict of Interest Statement}

Authors have nothing to disclose with regard to commercial support.

The study was based on data from the National Health Insurance Research Database provided by the National Health Insurance Administration, Ministry of Health and Welfare of Taiwan, and managed by the National Health Research Institutes of Taiwan. However, the interpretation and conclusions contained do not represent those of the National Health Insurance Administration, Ministry of Health and Welfare of Taiwan, or the National Health Research Institutes of Taiwan. Drs S-W Chen and T-S Chen had full access to all the data used in the study and take responsibility for the integrity of the data and accuracy of analysis. The authors thank Su-Wei Chang and Alfred Hsing-Fen Lin for their assistance in statistical analysis. 


\section{References}

1. Rastan AJ, Dege A, Mohr M, Doll N, Falk V, Walther T, et al. Early and late outcomes of 517 consecutive adult patients treated with extracorporeal membrane oxygenation for refractory postcardiotomy cardiogenic shock. J Thorac Cardiovasc Surg. 2010;139:302-11. 311.e1.

2. Wu MY, Lin PJ, Lee MY, Tsai FC, Chu JJ, Chang YS, et al. Using extracorporeal life support to resuscitate adult postcardiotomy cardiogenic shock: treatment strategies and predictors of short-term and midterm survival. Resuscitation. 2010;81:1111-6.

3. Abrams D, Combes A, Brodie D. Extracorporeal membrane oxygenation in cardiopulmonary disease in adults. J Am Coll Cardiol. 2014;63:2769-78.

4. Squiers JJ, Lima B, DiMaio JM. Contemporary extracorporeal membrane oxygenation therapy in adults: fundamental principles and systematic review of the evidence. J Thorac Cardiovasc Surg. 2016;152:20-32.

5. Saxena P, Neal J, Joyce LD, Greason KL, Schaff HV, Guru P, et al. Extracorporeal membrane oxygenation support in postcardiotomy elderly patients: the Mayo Clinic experience. Ann Thorac Surg. 2015;99:2053-60.

6. Wang JG, Han J, Jia YX, Zeng W, Hou XT, Meng X. Outcome of veno-arterial extracorporeal membrane oxygenation for patients undergoing valvular surgery. Plos One. 2013;8:e63924.

7. Slottosch I, Liakopoulos O, Kuhn E, Deppe AC, Scherner M, Madershahian N, et al. Outcomes after peripheral extracorporeal membrane oxygenation therapy for postcardiotomy cardiogenic shock: a single-center experience. J Surg Res. 2013;181:e47-55.

8. Aissaoui N, Luyt CE, Leprince P, Trouillet JL, Leger P, Pavie A, et al. Predictors of successful extracorporeal membrane oxygenation (ECMO) weaning after assistance for refractory cardiogenic shock. Intensive Care Med. 2011;37: $1738-45$.

9. Mazzeffi MA, Sanchez PG, Herr D, Krause E, Evans CF, Rector R, et al. Outcomes of extracorporeal cardiopulmonary resuscitation for refractory cardiac arrest in adult cardiac surgery patients. J Thorac Cardiovasc Surg. 2016;152: 1133-9.

10. McCarthy FH, McDermott KM, Kini V, Gutsche JT, Wald JW, Xie D, et al. Trends in U.S. extracorporeal membrane oxygenation use and outcomes: 20022012. Semin Thorac Cardiovasc Surg. 2015;27:81-8.

11. Li CL, Wang H, Jia M, Ma N, Meng X, Hou XT. The early dynamic behavior of lactate is linked to mortality in postcardiotomy patients with extracorporeal membrane oxygenation support: a retrospective observational study. J Thorac Cardiovasc Surg. 2015;149:1445-50.

12. Cheng R, Hachamovitch R, Kittleson M, Patel J, Arabia F, Moriguchi J, et al. Complications of extracorporeal membrane oxygenation for treatment of cardiogenic shock and cardiac arrest: a meta-analysis of 1,866 adult patients. Ann Thorac Surg. 2014;97:610-6.

13. Chen YC, Tsai FC, Fang JT, Yang CW. Acute kidney injury in adults receiving extracorporeal membrane oxygenation. J Formos Med Assoc. 2014;113:778-85.

14. Hsiao CC, Chang CH, Fan PC, Ho HT, Jenq CC, Kao KC, et al. Prognosis of patients with acute respiratory distress syndrome on extracorporeal membrane oxygenation: the impact of urine output on mortality. Ann Thorac Surg. 2014; 97:1939-44.

15. Wu MY, Lee MY, Lin CC, Chang YS, Tsai FC, Lin PJ. Resuscitation of nonpostcardiotomy cardiogenic shock or cardiac arrest with extracorporeal life support: the role of bridging to intervention. Resuscitation. 2012;83:976-81.
16. Hsu CP, Lee WC, Wei HM, Sung SH, Huang CY, Shih CC, et al. Extracorporeal membrane oxygenation use, expenditure, and outcomes in taiwan from 2000 to 2010. J Epidemiol. 2015;25:321-31.

17. Hsing AW, Ioannidis JP. Nationwide population science: lessons from the Taiwan National Health Insurance Research Database. JAMA Intern Med. 2015;175: 1527-9.

18. Cheng CL, Chien HC, Lee CH, Lin SJ, Yang YH. Validity of in-hospital mortality data among patients with acute myocardial infarction or stroke in National Health Insurance Research Database in Taiwan. Int J Cardiol. 2015;201:96-101.

19. Cheng CL, Lee CH, Chen PS, Li YH, Lin SJ, Yang YH. Validation of acute myocardial infarction cases in the National Health Insurance Research Database in Taiwan. J Epidemiol. 2014;24:500-7.

20. Wu CY, Chen YJ, Ho HJ, Hsu YC, Kuo KN, Wu MS, et al. Association between nucleoside analogues and risk of hepatitis B virus-related hepatocellular carcinoma recurrence following liver resection. JAMA. 2012;308:1906-14.

21. Rosenbaum PR, Rubin DB. The central role of the propensity score in observational studies for causal effects. Biometrika. 1983;70:41-55.

22. Austin PC. An introduction to propensity score methods for reducing the effects of confounding in observational studies. Multivariate Behav Res. 2011;46: $399-424$.

23. Fine JP, Gray RJ. A proportional hazards model for the subdistribution of a competing risk. J Am Stat Assoc. 1999;94:496-509.

24. Tarzia V, Bortolussi G, Bianco R, Buratto E, Bejko J, Carrozzini M, et al. Extracorporeal life support in cardiogenic shock: impact of acute versus chronic etiology on outcome. J Thorac Cardiovasc Surg. 2015;150:333-40.

25. Combes A, Leprince P, Luyt CE, Bonnet N, Trouillet JL, Leger P, et al. Outcomes and long-term quality-of-life of patients supported by extracorporeal membrane oxygenation for refractory cardiogenic shock. Crit Care Med. 2008;36:1404-11.

26. Anselmi A, Flecher E, Corbineau H, Langanay T, Le Bouquin V, Bedossa M, et al. Survival and quality of life after extracorporeal life support for refractory cardiac arrest: a case series. J Thorac Cardiovasc Surg. 2015;150:947-54.

27. Beurtheret S, Mordant P, Paoletti X, Marijon E, Celermajer DS, Leger P, et al. Emergency circulatory support in refractory cardiogenic shock patients in remote institutions: a pilot study (the cardiac-RESCUE program). Eur Heart J. 2013;34: 112-20.

28. Askenazi DJ, Selewski DT, Paden ML, Cooper DS, Bridges BC, Zappitelli M et al. Renal replacement therapy in critically ill patients receiving extracorporeal membrane oxygenation. Clin J Am Soc Nephrol. 2012;7:1328-36.

29. Chen H, Yu RG, Yin NN, Zhou JX. Combination of extracorporeal membrane oxygenation and continuous renal replacement therapy in critically ill patients: a systematic review. Crit Care. 2014; 18:675.

30. Hajjar LA, Vincent JL, Galas FR, Nakamura RE, Silva CM, Santos MH, et al Transfusion requirements after cardiac surgery: the TRACS randomized controlled trial. JAMA. 2010;304:1559-67.

31. Agerstrand CL, Burkart KM, Abrams DC, Bacchetta MD, Brodie D. Blood conservation in extracorporeal membrane oxygenation for acute respiratory distress syndrome. Ann Thorac Surg. 2015;99:590-5.

Key Words: extracorporeal membrane oxygenation, cardiac surgery, postcardiotomy shock, prognosis 

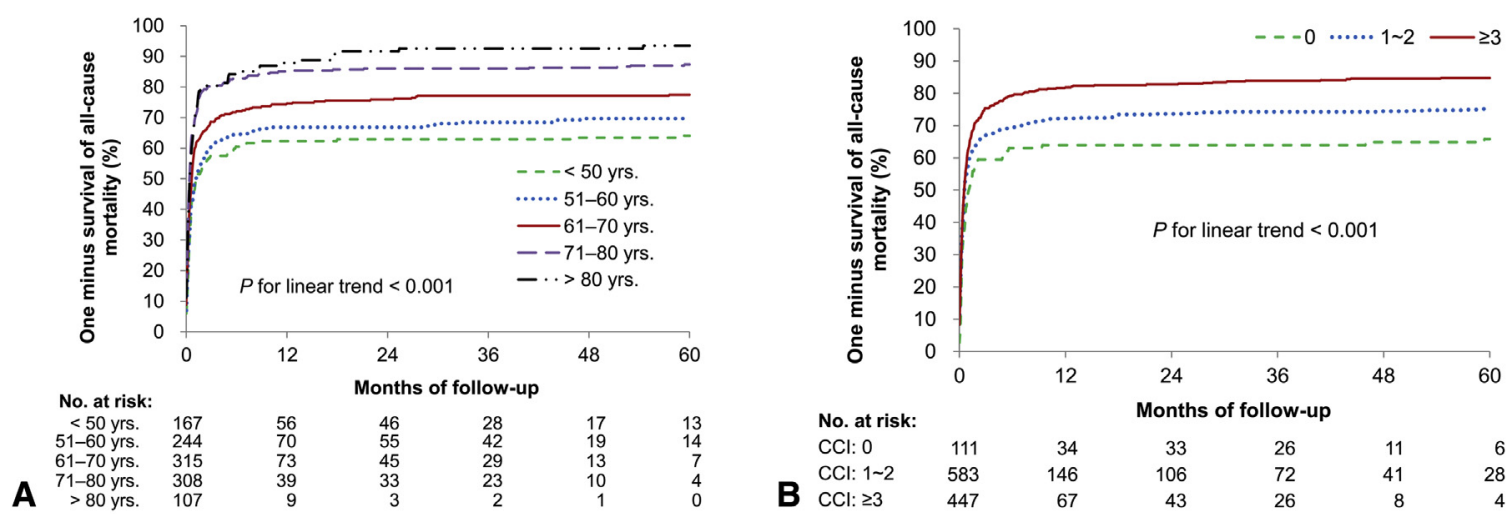

\begin{tabular}{rrrrrr}
\multicolumn{7}{c}{ Months of follow-up } \\
111 & 34 & 33 & 26 & 11 & 6 \\
583 & 146 & 106 & 72 & 41 & 28 \\
447 & 67 & 43 & 26 & 8 & 4
\end{tabular}

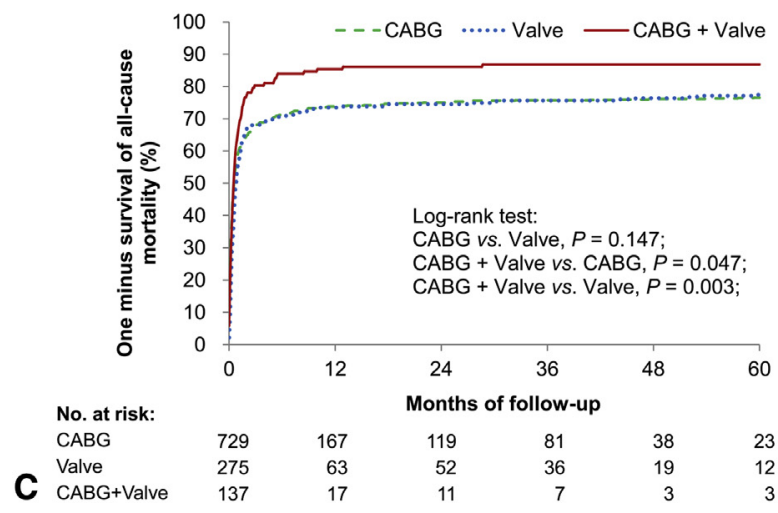

FIGURE E1. Kaplan-Meier survival curves of all-cause mortality in patients who received ECMO for PCS stratified by a predefined subgroup of (A) age group, (B) CCI score, and (C) surgery type. CCI, Charlson comorbidity index; $C A B G$, coronary artery bypass grafting.

TABLE E1. International Classification of Diseases, Ninth Revision, Clinical Modification code used for diagnosis in the current study

\begin{tabular}{ll}
\hline \multicolumn{1}{c}{ Variable } & \multicolumn{1}{c}{ Code } \\
\hline Diabetes mellitus & $250 . \mathrm{xx}$ \\
Hypertension & $401 . \mathrm{xx}-405 . \mathrm{xx}$ \\
Congestive heart failure & $428 . \mathrm{xx}$ \\
Coronary artery disease & $410 . \mathrm{xx}-414 . \mathrm{xx}$ \\
Prior myocardial infarction & $410 . \mathrm{xx}, 412 . \mathrm{xx}$ \\
Acute myocardial infarction & $410 . \mathrm{xx}$ \\
Peripheral arterial disease & $440.0,440.2 \mathrm{x}, 440.3 \mathrm{x}, 440.4,440.9$, \\
& $443.9,444.2,444.22,444.8$, \\
& $444.81,445.0,445.02,250.7 \mathrm{x}$, \\
& $707.1 \mathrm{x}$ \\
Prior stroke & $430 . \mathrm{xx}-437 . \mathrm{xx}$ \\
Chronic kidney disease & $585 . \mathrm{xx}$ \\
Chronic obstructive pulmonary & $490 . \mathrm{xx}-496 . \mathrm{xx}$ \\
$\quad$ disease & $571.5,571.6$ \\
Liver cirrhosis & $140 . \mathrm{xx}-208 . \mathrm{xx}$ \\
Malignancy & $430 . \mathrm{xx}-432 . \mathrm{xx}$ \\
Hemorrhagic stroke & $433 . \mathrm{xx}-435 . \mathrm{xx}$ \\
Ischemic stroke & $038 . \mathrm{xx}, 9985$ \\
Postoperative infection &
\end{tabular}


TABLE E2. Factors associated with in-hospital survival in the ECMO $\operatorname{group}(\mathbf{n}=1141)$

\begin{tabular}{lcr}
\hline \multicolumn{1}{c}{ Variable } & Odds ratio (95\% CI) & \multicolumn{1}{c}{$\boldsymbol{P}$} \\
\hline Age, y & $0.97(0.96-0.98)$ & $<.001$ \\
Female gender & $0.91(0.68-1.22)$ & .528 \\
Diabetes mellitus & $1.14(0.86-1.52)$ & .349 \\
Hypertension & $1.41(1.06-1.87)$ & .018 \\
Congestive heart failure & $0.58(0.42-0.81)$ & .001 \\
Coronary artery disease & $1.38(0.83-2.28)$ & .213 \\
Prior myocardial infarction & $0.96(0.69-1.34)$ & .823 \\
Peripheral arterial disease & $1.20(0.70-2.08)$ & .509 \\
Prior stroke & $0.74(0.51-1.07)$ & .105 \\
Chronic kidney disease or dialysis & $0.66(0.43-1.03)$ & .066 \\
COPD & $0.97(0.59-1.60)$ & .918 \\
Liver cirrhosis & $0.28(0.08-0.97)$ & .045 \\
Malignancy & $1.41(0.71-2.82)$ & .330 \\
Surgical type & & \\
$\quad$ CABG alone & Reference & N/A \\
Valve alone & $1.42(0.89-2.27)$ & .144 \\
CABG + Valve & $0.97(0.64-1.45)$ & .877 \\
\hline
\end{tabular}

$C I$, Confidence interval; $C O P D$, chronic obstructive pulmonary disease; $C A B G$, coronary artery bypass grafting; $N / A$, not applicable.

TABLE E3. Factors associated with 1-year survival in the ECMO group $(\mathbf{n}=1141)$

\begin{tabular}{lcr}
\hline \multicolumn{1}{c}{ Variable } & Odds ratio $(\mathbf{9 5} \% \mathbf{C I})$ & $\boldsymbol{P}$ \\
\hline Age, y & $0.97(0.95-0.98)$ & $<.001$ \\
Female gender & $0.95(0.68-1.32)$ & .760 \\
Diabetes mellitus & $1.06(0.77-1.46)$ & .709 \\
Hypertension & $1.29(0.93-1.77)$ & .122 \\
Congestive heart failure & $0.59(0.41-0.86)$ & .006 \\
Coronary artery disease & $1.13(0.63-2.00)$ & .686 \\
Prior myocardial infarction & $1.17(0.80-1.69)$ & .419 \\
Peripheral arterial disease & $1.02(0.53-1.93)$ & .962 \\
Prior Stroke & $0.91(0.60-1.39)$ & .666 \\
Chronic kidney disease or dialysis & $0.45(0.26-0.79)$ & .006 \\
COPD & $0.78(0.42-1.44)$ & .420 \\
Liver cirrhosis & $0.37(0.08-1.67)$ & .197 \\
Malignancy & $0.84(0.35-2.01)$ & .703 \\
Surgical type & & \\
$\quad$ CABG alone & Reference & N/A \\
$\quad$ Valve alone & $1.13(0.66-1.94)$ & .648 \\
CABG + Valve & $0.59(0.35-1.001)$ & .051 \\
\hline CII Confidence interva; $C O P D$, chronic obstructive pulmonary disese; $C A B C$.
\end{tabular}

$C I$, Confidence interval; $C O P D$, chronic obstructive pulmonary disease; $C A B G$, coronary artery bypass grafting; $N / A$, not applicable. 